\title{
Insights on Bridging Stent Grafts in Fenestrated and Branched Aortic Endografting
}

\author{
William J. Yoon ${ }^{1,2}$, Victor M. Rodriguez ${ }^{1,3}$, and Cheong Jun Lee $^{4}$ \\ ${ }^{1}$ Division of Vascular Surgery, Department of Surgery, University of California-Davis, Sacramento, CA, USA, ${ }^{2}$ Section of Vascular \\ Surgery, Department of Surgical Sciences, Uppsala University, Uppsala, Sweden, ${ }^{3}$ Division of Cardiothoracic Surgery, Department of \\ Surgery, University of California-Davis, Sacramento, CA, ${ }^{4}$ Division of Vascular Surgery, Department of Surgery, NorthShore University \\ HealthSystem, University of Chicago Pritzker School of Medicine, Evanston, IL, USA
}

\begin{abstract}
Major branches of the aortic arch and visceral aorta pose a particular challenge for endovascular repair of aneurysms involving these regions. To preserve perfusion through these essential branches, fenestrated and branched endografts have been used. Current fenestrated and branched aortic endografts have evolved into modular devices in which the aortic main body provides appropriate access to the target branch vessel either through reinforced fenestrations or directional cuffs as the hinge point for bridging stent grafts (BSGs). BSGs are used to connect the aortic main body and target branch vessel, and must provide both unhindered flow and a seal. Appropriate selection of BSG for target vessels in branched and fenestrated endovascular aortic repair is critical for technical success and durability. At present, there are no dedicated devices for use as BSGs, and a variety of stent grafts are currently used off-label. In this report, we review the available published series on the performance of presently available BSGs in relation to their design and selection.
\end{abstract}

Key Words: Branched, Bridging, Endovascular, Fenestrated, Stent
Received March 25, 2021

Revised May 27, 2021

Accepted May 28, 2021

Published on June 17, 2021

Corresponding author: William J. Yoon

Division of Vascular Surgery, Department of Surgery, University of California-Davis Medical Center, 2335 Stockton Blvd., NAOB 5016 Sacramento, CA 95817, USA Tel: 1-916-734-2028

Fax: 1-916-734-2030

E-mail: william.yoon@protonmail.com https://orcid.org/0000-0002-9413-0396

Copyright (c) 2021 The Korean Society for Vascular Surgery

This is an Open Access article distributed under the terms of the Creative Commons Attribution Non-Commercial License (http://creativecommons.org/licenses/by-nc/4.0) which permits unrestricted non-commercial use, distribution, and reproduction in any medium, provided the original work is properly cited.

Vasc Specialist Int • https://doi.org/10.5758/vsi.210025

\section{INTRODUCTION}

Despite considerable evolution in endovascular repair of aortic aneurysms (EVAR), aortic pathology involving aortic branches remains challenging. Currently, no standardized endovascular aortic device exists to meet the complexity and anatomic variability encountered in these repairs. To preserve perfusion through essential aortic branches, fenestrated and branched endografts have been used since the late 1990s. Park et al. [1] in 1996 performed the first successful fenestrated EVAR (fEVAR) to treat two patients with abdominal aortic aneurysms (AAAs) involving visceral aortic branches. In 1999, a covered aortic stent with a fenestration was proposed by Browne et al. [2]. Contemporaneous with this proposal, the feasibility of using branched stent grafts was illustrated by lnoue et al. [3] in the treatment of both the aortic arch and thoracoabdominal aorta [4].

The primary goal of these complex endovascular aortic repairs is to extend the proximal landing zone for the main body device in a healthy segment of the aorta to achieve appropriate proximal seal. The challenge of these repairs is predicated on the number of visceral branches needing to be incorporated and preserved in order to achieve the ideal proximal seal zone for the reconstruction. Technical experience and improved device design over time have allowed physicians to overcome multiple branches. Most recently, Eleshra et al. [5] reported successful management of a patient with variant visceral vessel anatomy (bilateral double renal arteries with both common hepatic and splenic arteries originating from the aorta) with a stent graft with seven 
branches.

The first branched stent grafts Inoue et al. [3] envisioned were unibody constructions without the ability to change the length and position of the branch at the time of implantation. Such designs pose restrictive utility with geometric variability that is common with visceral branch anatomy. To overcome anatomic limitations, physician modified fEVAR and branched EVAR (bEVAR) have been predominantly performed in modular fashion [6]. With a modular strategy, the aortic main body component provides access to the target branch vessel either through fenestrations or cuffs. Bridging stent grafts (BSGs) are then used to connect the aortic main body and the target branch vessel. In the modular construct, BSGs must provide both favorable and durable perfusion to the target vessel while providing a proper component seal.

Inherent with aneurysm exclusion, the technical and clinical success of fEVAR and bEVAR ( $f / b E V A R$ ) is driven by target vessel patency. In a study by Panuccio et al. [7] reporting the findings of 523 target vessels in 150 patients treated with $\mathrm{f} / \mathrm{bEVAR}$, use of a BSG was the only independent risk factor for re-intervention (hazard ratio, 3.5; 95\% confidence interval [Cl], 1.3-9.9; $\mathrm{P}=0.02$ ). In a more recent study by Oderich et al. [8] reporting on outcomes of fenestrated and branched endografts, all reinterventions after $\mathrm{f} /$ bEVAR were branch stent revisions. Taken together, it can be inferred that selection of an appropriate BSG plays a critical role on the durability of the repair. A variety of devices are currently used as BSGs (Table 1); however, not one stent graft has a dedicated indication for complex aortic repairs.

\section{CONTROVERSY WITH RESPECT TO SELECTION OF FENESTRATIONS OR DIRECTIONAL BRANCHES}

During the past decade, stent graft technology has evolved rapidly to accommodate a wide variety of main body endograft structural designs that may incorporate both fenestrations and directional cuffs. A cuff is a short branch attached to the aortic main body component for the attachment of a BSG. The length of the cuff provides an appreciable overlap between the BSG and the attachment site. The resultant sealing and stabilization minimize the risk of type 111 endoleak and the risk of disconnection $[9,10]$.

On the other hand, a fenestration is a hole in the fabric of aortic main body component. Reinforced with a circumferential nitinol wire and customized to the size of the target artery, fenestrations are positioned adjacent to the target aortic branch artery orifice. These fenestrations are then fixed in position over the ostia by deploying BSGs through them and into the target arteries. This creates a seal between the BSG and the fenestration. But there is no overlap and hence the potential for type 111 endoleaks around fenestration sites and disconnection risk remains. Accordingly, to ensure stability as well as to prevent endoleaks, the diameter of the BSG within the fenestration must be properly oversized [11]. To further secure these BSGs in place after deployment, a significant portion of the BSG is allowed to protrude into the aortic main body component. The protruding portion is then flared with a larger balloon on the inside of the aortic main component, forming a funnel-shaped conduit for blood flow into the target arteries (Fig. 1) [12,13].

The hemodynamic impact of a flared renal stent on the performance of fenestrated endografts was analyzed by Kandail et al. [13] using computational fluid dynamics. The results showed flaring does not compromise the renal flow, but it causes flow disturbance at the renal ostia, making these regions potentially susceptible to thrombus formation. Still, by reducing the dilation angle (due to flaring) or protrusion length, the risk of thrombosis can be alleviated, thereby improving the durability of flared BSGs [13].

There is still controversy with respect to which type is best. Some experts advocate fenestrations, while others prefer directional branches for visceral artery incorpora-

Table 1. Existing bridging stents for fenestrated and branched endografts

\begin{tabular}{cll}
\hline Stent type & \multicolumn{1}{c}{ Product name } & \multicolumn{1}{c}{ Manufacturer } \\
\hline Balloon-expandable & iCast/Advanta V12 & Maquet Getinge Group, Hirrlingen, Germany \\
& BeGraft \& BeGraft Plus & Bentley InnoMed GmbH, Hechingen, Germany \\
& E-ventus & Jotec GmbH, Hechingen, Germany \\
& Viabahn VBX & W.L. Gore and Associates Inc., Flagstaff, AZ, USA \\
& LifeStream & CR Bard Inc., Tempe, AZ, USA \\
Self-expandable & Fluency Plus Flair & CR Bard Inc., Tempe, AZ, USA \\
& Viabahn & W. L. Gore and Associates Inc., Flagstaff, AZ, USA \\
& Covera & CR Bard Inc., Tempe, AZ, USA \\
& Wallgraft & Boston Scientific Corp., Watertown, MA, USA
\end{tabular}



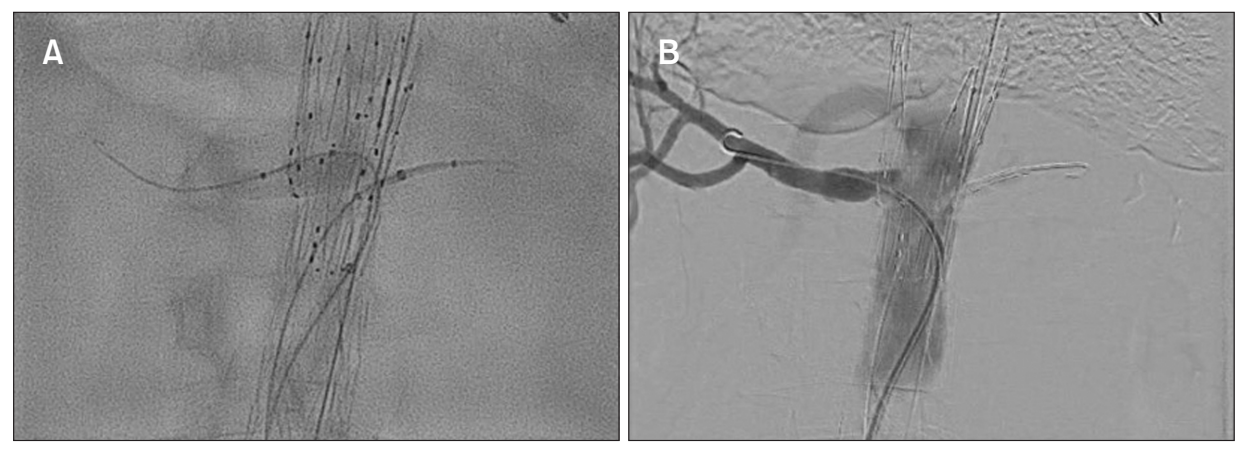

Fig. 1. Fenestration. (A) Bridging stent flaring. (B) Completion angiogram post flaring.

tion. Chuter et al. [14] in 2011 reported their transition from using a fenestration as a BSG attachment point to cuffed joint type in their study with 28 patients who underwent thoracoabdominal endovascular aneurysm repair. The reasons for their preference for cuffs were two-fold: (1) the ability to implant the device without a high degree of precision and (2) the option of using self-expandable BSGs in a cuffed stent graft. On the other side, in a series which incorporated 523 visceral vessels in 158 patients (140 in juxtarenal AAAs, 165 in pararenal AAAs, and 218 in thoraco AAAs [TAAAs]), Pini et al. [15] evaluated the visceral vessel loss according to the type of revascularization performed (fenestrations vs. directional branches). The overall perioperative visceral vessel loss in this study was 20 (3.8\%), and the branches resulted in greater perioperative visceral vessel loss compared with fenestrations (9\% [11/122] vs. $2 \%$ [9/401], $P=0.0001)$. Furthermore, a significant visceral vessel loss difference between the branches and fenestrations was identified selectively only for the renal arteries (21\% [11/52] vs. $2.5 \%$ [6/224], $\mathrm{P}=0.001)$. Similar findings were obtained in a Nuremberg study reporting outcomes of 347 patients treated with f/bEVAR for TAAAs in which fenestrations only were used in $108(31.1 \%)$ patients, directional branches only in $104(30.0 \%)$ patients, and a stent-graft with a combination of fenestrations and branches in 135 (38.9\%) patients, target vessel patency at 3 years was $98 \%$ for vessels targeted with fenestrations and 92\% for vessels targeted with branches $(\mathrm{P}=0.009)$ [16].

Whilst the choice between fenestration and branches has been the subject of debate, Mastracci et al. [17] reviewed 650 patients who underwent EVAR with branched or fenestrated devices. In this series, it was noted that the superior mesenteric and celiac arteries both arise from the anterior aorta and course downward, whereas the renal arteries are small and frequently oriented cranially or directed laterally. It was on this account that Mastracci et al. [17] strongly suggested fenestrations only for renal arteries and branches for visceral arteries. Similarly, in a series by Panuccio et al. [7] in that the joint type was $48 \%$ fenestration vs 52\% cuffs, cuffs showed a statistically significant association with reintervention (hazard ratio, 3.5; 95\% $\mathrm{Cl}, 1.3-9.9 ; \mathrm{P}=0.02$ ). Nonetheless, the authors stated that the higher reintervention rate observed could be the result of selection bias, and that their preference has been to select fenestrated device only when the aortic main component has contact with the aortic wall and cuffed devices for branch vessels that originate from a large aortic lumen.

All in all, despite ongoing controversy surrounding selection of fenestrations or directional branches, most experts agree that directional branches should be used for downward vessels that originate from large aortic lumen with a steeper take-off angle and when there is a larger gap to be bridged, whereas fenestrations should be used for vessels that originate from the sealing zone with a close to $90^{\circ}$ take-off from the aorta and when the main aortic body at the level of the target vessel is adjacent to the aortic wall [7,16-19].

\section{RENAL ARTERY STENTING VS. STENTING OF THE SUPERIOR MESENTERIC ARTERY AND/OR CELIAC ARTERY}

Loss of target visceral branch patency is one of the most important complications of $\mathrm{f} / \mathrm{bEVAR}$. Several studies have shown that risk factors for BSG instability include renal artery stent grafts vs. superior mesenteric artery (SMA) and celiac trunk (CT) stent grafts [7,17,20,21]. In the experience reported by Panuccio et al. [7], incorporating 523 target vessels (104 CT, 140 SMA, 138 right renal artery, 134 left renal artery), the renal artery as the target vessel showed a statistically significant association with peri-operative vessel related events (odds ratio, 13.2; 95\% Cl, 1.5-118.4; $\mathrm{P}=0.02$ ). Interestingly, in this study, the Kaplan-Meier curve for freedom from occlusion comparing different type of target vessel for BSG showed no statistically significant differences, suggesting the type of target vessel may no longer represent a significant risk factor in the long-term. Reilly et al. [19] also evaluated 306 branches in 81 patients undergoing TAAA repair using the caudally directed cuff technique and reported renal artery occlusion as the most 
common form of branch failure (13 renal vs. 1 SMA vs. 2 CT occlusions/stenoses). Similarly, Mastracci et al. [20] investigated 940 vessels that underwent stent graft for treatment of TAAA that used only branches to mate with visceral and renal vessels. Their results showed that the event rate in renal branches $(35 / 437,8 \%)$ was higher than that of visceral branches $(8 / 443,1.8 \%)$, and there was a statistically significant difference between renal and visceral artery occlusions (hazard ratio, 3.51; $\mathrm{P}=0.001$ ).

As depicted in the aforementioned studies, renal arteries carry a higher rate of loss. Some known factors for branch failure include small vessel diameter, tortuous anatomy, and upward vessel angulation [17,22]. Compared with the SMA and CT, renal arteries have a smaller diameter, acute angulation, and are more mobile and tortuous [7,19]. In view of these anatomical characteristics, it can be postulated that some of the renal complications might have been on account of poor selection of the method of incorporation rather than the BSG itself. Hence, careful analysis of renal take-off angles must be taken into consideration when selecting the method of renal incorporation.

\section{WHICH TYPE OF BRIDGING STENT SHOULD BE USED: COVERED STENTS VS. UNCOVERED STENTS}

The implantation of stent graft causes stretching of the vessel wall at the site of implantation. This process may disrupt the intima, resulting in the initiation of neointimal proliferation and hyperplasia, which, in turn, can lead to stenosis [23,24]. Studies have shown that covered stents can decrease the rate of stenosis. Mohabbat et al. [25] reviewed a total of 518 renal arteries that were treated with uncovered $(n=287)$ or covered stents $(n=231)$. The estimated freedom from stenosis, in this study, at 12, 24, and 36 months were 95\%, 92\%, and 89\% for uncovered stents, and 98\%, $97 \%$, and $95 \%$ for covered stents $(P=0.04)$. Grimme et al. [26] also reported that renal artery stenosis occurred significantly more with uncovered stents than with covered stents $(\mathrm{P}=0.04)$. In a study by Oderich et al. [27], in that outcomes for covered stents were compared with those for uncovered stents in 225 patients with chronic mesenteric ischemia, covered stents outperformed uncovered stents with less restenosis and better patency rates. A possible explanation for these observed superior patency rates is thought to be that covered stents act as a physical barrier to hamper intimal hyperplasia [28].

Additionally, studies also reported fewer fractures with covered stents than with uncovered stents [26,29]. A possible reason for this observation could be that the polytetrafluoroethylene (PTFE) layers in the covered stents create high radial strength by connecting the stent struts, wherefore covered stents might be less prone to fatigue fracture $[29,30]$. For the aforementioned reasons, covered stents are preferred in branch endografting.

The current indications for use of uncovered stents during f/bEVAR are limited to (1) extending distal sealing of covered stents to prevent the kinking of visceral stents; (2) treating endoleaks originating from the distal seal of visceral stents; and (3) preventing distal outflow obstructions [29]. Even so, there have been concerns over the use of this relining concept as uncovered stents are considered to be associated with loss of visceral branch patency. Moreover, studies do not support this concept. In a series incorporating 442 target vessels, Khoury et al. [30] evaluated whether the use of distal extension with uncovered stents influences vessel patency. They found that at 12 months, the overall primary patency rate was $86 \%$ in the distal extension group vs. $93 \%$ when covered stents only $(P=0.8)$, and the rate of branch-related reinterventions was 9\% and 15\%, respectively $(\mathrm{P}=0.5)$, suggesting the use of distal uncovered stents to prevent kinks was not associated with decreased early branch patency [30]. Panuccio et al. [7] also failed to confirm the efficacy of the relining strategy as crimping of the BSG was the most frequent source of problems (type 1 endoleak and restenosis of the BSGs) in their series, despite the extensive use of relining stents (64.6\%). As a possible explanation for the crimping of the BSG, Panuccio et al. [7] hypothesized that the pinching load on the BSG caused by the remodeling of the aorta and the vessel pulsatility may induce plastic deformation in the long term.

\section{WHICH TYPE OF BRIDGING STENT SHOULD BE USED: BALLOON- OR SELF-EXPANDABLE STENT}

Selection of the BSG for f/bEVAR has been controversial among users of the technology from its inception. Currently, two types of covered stents are commercially available for branch endografting: balloon-expandable (BE) and self-expandable (SE). Each type has its own advantages and disadvantages.

Advocates of SE stents quote their flexibility and conformability as the justification for SE superiority over BE stents [20]. And because of these characteristics, SE BSGs have been preferred for directional branches (Fig. 2). In such circumstances, where the vessel is tortuous and where morphology involves proximal stenosis and luminal irregularities related to atherosclerotic disease, SE stents are likely to perform better than BE stents [7,17,22]. Important drawbacks of SE BSG include less precise deployment, poor trackability, and low variability of length available [7]. Of 
note, the continuous and radially directed expanding force exerted by SE stents after deployment may lead to a modification of the natural target vessel anatomy.

On the other hand, BE stents are traditionally used for transversely oriented vessels aligned by fenestrations because they are rigid, available in shorter length, and ability to flare [22]. Proponents of BE stents also cite their smaller delivery system, precise deployment, prevention of embolism by entrapment of debris, and less risk of arterial disruption as added advantages [20,22]. The recently introduced BE VBX (W. L. Gore \& Associates Inc., Flagstaff, AZ, USA) uses expanded PTFE to connect the stent rows of the VBX instead of connecting bars. This design has been shown to increase flexibility that matches the flexibility of SE stents (Fig. 3). Other newly available devices, BeGraft (Bentley InnoMed GmbH, Hechingen, Germany) and E-

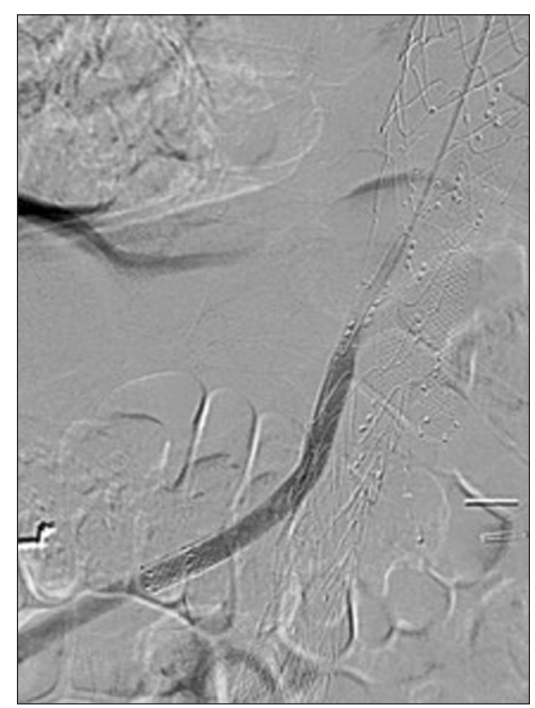

Fig. 2. Directional branch with self-expandable stent.
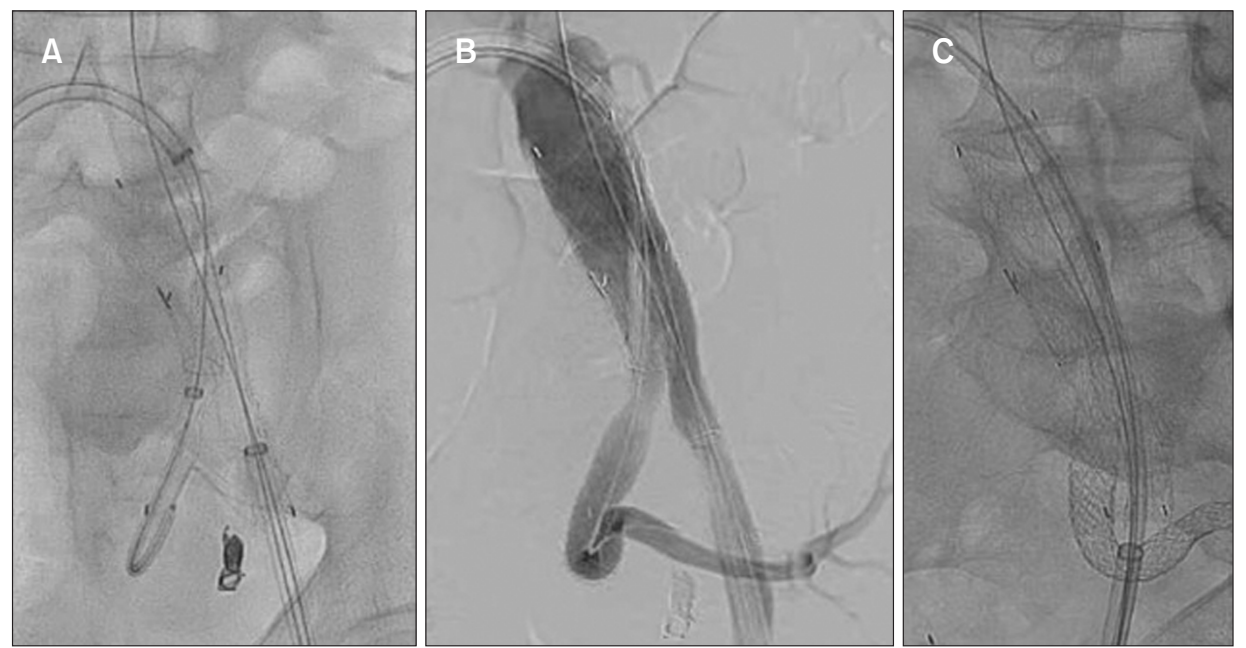

Fig. 3. (A) Flexibility of the balloon expandable stent (Viabahn VBX) during delivery. (B) Completion angiogram post deployment. (C) Oblique view of the stent. 
cardiac cycles is substantial [31-33]. Thus, aortic branch artery motion during the respiratory and cardiac cycle is significant. As such, BSGs connected into aortic branch vessels may be affected by the motion of the involved artery branches. Specifically, cyclical stress and strain deteriorate not only stent-graft attachment, but also stent-graft structure, which may lead to complications such as kink, fracture, and migration. Muhs et al. [34,35], using dynamic cine-computed tomography angiography with a 64-slice scanner, studied the natural renal artery motion during cardiac cycles in patients with abdominal aortic aneurysm and how the implantation of fenestrated endografts with renal BSGs may distort this movement. Their results showed that renal stents inhibited proximal renal artery motion, resulting in a $31 \%$ decrease in maximal motion $(\mathrm{P}<0.05)$. As the BSG holds the branch vessel in alignment, translation of the branch artery motion occurs along the BSG, focusing the force translated at the distal end of the BSG instead of being normally distributed over the length of the branch artery. Additional to these kinetic forces, there is a compliance mismatch between the BSG and native branch artery [36,37]. Together, these kinetic force and compliance mismatch subject BSGs to repetitive stress, which, in turn, may make these BSGs vulnerable not only to fracture, but also to the development of intimal hyperplasia [37].

\section{CONCLUSION}

Fenestrated and branched endografts have greatly expanded vascular surgeons' armamentarium for the management of complex aortic disease. The bridging stent that connects the aortic main component to the target vessel is a key component of $f / b E V A R$. Yet there is no agreement as to ideal bridging stent for target branches. This lack of consensus has led to vascular surgeon using a wide combination of SE or BE covered stents. Important prerequisites for BSGs include flexibility and resistance to mechanical as well as compliance mismatch stress in connecting the stiff aortic main body with moving target arteries. A dedicated device may improve the performance of bridging stents.

\section{CONFLICTS OF INTEREST}

William J. Yoon is a paid consultant to Cook Medical LLC and W. L. Gore \& Associates Inc. The other authors have nothing to disclose.

\section{ORCID}

\author{
William J. Yoon \\ https://orcid.org/0000-0002-9413-0396 \\ Victor M. Rodriguez \\ https://orcid.org/0000-0002-8495-0171 \\ Cheong Jun Lee \\ https://orcid.org/0000-0003-1439-5659
}

\section{AUTHOR CONTRIBUTIONS}

Concept and design: WJY. Analysis and interpretation: none. Data collection: none. Writing the article: WJY, VMR, CJL. Critical revision of the article: WJY, VMR, CJL. Final approval of the article: WJY, VMR, CJL. Statistical analysis: none. Obtained funding: none. Overall responsibility: WJY.

\section{REFERENCES}

1) Park JH, Chung JW, Choo IW, Kim SJ, Lee JY, Han MC. Fenestrated stent-grafts for preserving visceral arterial branches in the treatment of abdominal aortic aneurysms: preliminary experience. J Vasc Interv Radiol 1996;7:819-823.

2) Browne TF, Hartley D, Purchas S, Rosenberg M, Van Schie G, LawrenceBrown M. A fenestrated covered suprarenal aortic stent. Eur J Vasc Endovasc Surg 1999;18:445-449.

3) Inoue K, Hosokawa H, Iwase T, Sato M, Yoshida Y, Ueno K, et al. Aortic arch reconstruction by transluminally placed endovascular branched stent graft. Circulation 1999;100(19 Suppl):11316-11321.

4) Hosokawa H, Iwase T, Sato M, Yoshida Y, Ueno K, Tamaki S, et al. Successful endovascular repair of juxtarenal and suprarenal aortic aneurysms with a branched stent graft. J Vasc Surg 2001;33:1087-1092.

5) Eleshra A, Spanos K, Panuccio G, Gronert C, Rohlffs F, Kölbel T. Endovascular repair using a 7-branch stentgraft for a thoracoabdominal aortic aneurysm with variant renovisceral artery anatomy. J Endovasc Ther 2020;27:231-236.

6) Reilly LM, Chuter TA. Endovascular repair of thoracoabdominal aneurysms: design options, device construct, patient selection and complications. J Cardiovasc Surg (Torino) 2009;50:447460.

7) Panuccio G, Bisdas T, Berekoven B, Torsello G, Austermann M. Performance of bridging stent grafts in fenestrated and branched aortic endografting. Eur J Vasc Endovasc Surg 
2015;50:60-70.

8) Oderich GS, Ribeiro M, Hofer J, Wigham J, Cha S, Chini J, et al. Prospective, nonrandomized study to evaluate endovascular repair of pararenal and thoracoabdominal aortic aneurysms using fenestrated-branched endografts based on supraceliac sealing zones. J Vasc Surg 2017;65:12491259.e10.

9) Oderich GS, Fatima J, Gloviczki P. Stent graft modification with minicuff reinforced fenestrations for urgent repair of thoracoabdominal aortic aneurysms. J Vasc Surg 2011;54:15221526.

10) Troisi N, Donas KP, Austermann M, Tessarek J, Umscheid T, Torsello G. Secondary procedures after aortic aneurysm repair with fenestrated and branched endografts. J Endovasc Ther 2011;18:146-153.

11) Georgiadis GS, van Herwaarden JA, Antoniou GA, Giannoukas AD, Lazarides MK, Moll FL. Fenestrated stent grafts for the treatment of complex aortic aneurysm disease: a mature treatment paradigm. Vasc Med 2016;21:223-238.

12) Verhoeven EL, Katsargyris A, Fernandes e Fernandes R, Bracale UM, Houthoofd S, Maleux G. Practical points of attention beyond instructions for use with the Zenith fenestrated stent graft. J Vasc Surg 2014;60:246-252.

13) Kandail H, Hamady M, Xu XY. Effect of a flared renal stent on the performance of fenestrated stent-grafts at rest and exercise conditions. J Endovasc Ther 2016;23:809-820.

14) Chuter TA, Hiramoto JS, Park KH, Reilly LM. The transition from custommade to standardized multibranched thoracoabdominal aortic stent grafts. J Vasc Surg 2011;54:660-667; discussion 667-668.

15) Pini R, Faggioli G, Gallitto E, Mascoli C, Fenelli C, Ancetti S, et al. The different effect of branches and fenestrations on early and long-term visceral vessel patency in complex aortic endovascular repair. J Vasc Surg 2020;71:11281134.

16) Katsargyris A, Marques de Marino $P$, Verhoeven EL. Graft design and selection of fenestrations vs. branches for renal and mesenteric incorporation in endovascular treatment of pararenal and thoracoabdominal aortic aneurysms. J Cardiovasc Surg (Torino) 2019;60:35-40.

17) Mastracci TM, Greenberg RK, Eagleton MJ, Hernandez AV. Durability of branches in branched and fenestrated endografts. J Vasc Surg 2013;57:926933; discussion 933.

18) Chuter T, Greenberg RK. Standardized off-the-shelf components for multibranched endovascular repair of thoracoabdominal aortic aneurysms. Perspect Vasc Surg Endovasc Ther 2011;23:195-201.

19) Reilly LM, Rapp JH, Grenon SM, Hiramoto JS, Sobel J, Chuter TA. Efficacy and durability of endovascular thoracoabdominal aortic aneurysm repair using the caudally directed cuff technique. J Vasc Surg 2012;56:53-63; discussion 63-64.

20) Mastracci TM, Carrell T, Constantinou J, Dias N, Martin-Gonzalez T, Katsargyris A, et al. Editor's choice - effect of branch stent choice on branchrelated outcomes in complex aortic repair. Eur J Vasc Endovasc Surg 2016;51:536-542.

21) Martin-Gonzalez T, Mastracci T, Carrell T, Constantinou J, Dias N, Katsargyris A, et al. Mid-term outcomes of renal branches versus renal fenestrations for thoraco-abdominal aneurysm repair. Eur J Vasc Endovasc Surg 2016;52:141-148.

22) Tenorio ER, Kärkkäinen JM, Mendes BC, DeMartino RR, Macedo TA, Diderrich $A$, et al. Outcomes of directional branches using self-expandable or balloon-expandable stent grafts during endovascular repair of thoracoabdominal aortic aneurysms. J Vasc Surg 2020;71:1489-1502.e6.
23) Yucel S, Bahcivan M, Gol MK, Erenler BH, Kolbakir F, Keceligil HT. Reduced intimal hyperplasia in rabbits via medical therapy after carotid venous bypass. Tex Heart Inst J 2009;36:387392.

24) Heidari M, Mandato CA, Lehoux S. Vascular smooth muscle cell phenotypic modulation and the extracellular matrix. Artery Res 2015;9:14-18.

25) Mohabbat W, Greenberg RK, Mastracci TM, Cury M, Morales JP, Hernandez AV. Revised duplex criteria and outcomes for renal stents and stent grafts following endovascular repair of juxtarenal and thoracoabdominal aneurysms. J Vasc Surg 2009;49:827837; discussion 837.

26) Grimme FA, Zeebregts CJ, Verhoeven EL, Bekkema F, Reijnen MM, Tielliu IF. Visceral stent patency in fenestrated stent grafting for abdominal aortic aneurysm repair. J Vasc Surg 2014;59:298-306.

27) Oderich GS, Erdoes LS, Lesar C, Mendes BC, Gloviczki P, Cha S, et al. Comparison of covered stents versus bare metal stents for treatment of chronic atherosclerotic mesenteric arterial disease. J Vasc Surg 2013;58:1316-1323.

28) Takano M, Yamamoto M, Inami S, Xie Y, Murakami D, Okamatsu K, et al. Delayed endothelialization after polytetrafluoroethylene-covered stent implantation for coronary aneurysm. Circ J 2009;73:190-193.

29) Greenberg R, Eagleton M, Mastracci T. Branched endografts for thoracoabdominal aneurysms. J Thorac Cardiovasc Surg 2010;140(6 Suppl):S171S178.

30) Khoury MK, Timaran DE, Knowles M, Timaran $\mathrm{CH}$. Visceral stent patency after fenestrated endovascular aneurysm repair using bare-metal stent extensions versus covered stents only. J Vasc Surg 2020;71:23-29.

31) Teutelink A, Muhs BE, Vincken KL, Bartels LW, Cornelissen SA, van Herwaarden JA, et al. Use of dynamic 
computed tomography to evaluate pre- and postoperative aortic changes in AAA patients undergoing endovascular aneurysm repair. J Endovasc Ther 2007;14:44-49.

32) Draney MT, Zarins CK, Taylor CA. Three-dimensional analysis of renal artery bending motion during respiration. J Endovasc Ther 2005;12:380386.

33) Kaandorp DW, Vasbinder GB, de Haan MW, Kemerink GJ, van Engelshoven JM. Motion of the proximal renal artery during the cardiac cycle. J Magn
Reson Imaging 2000;12:924-928.

34) Muhs BE, Vincken KL, Teutelink A, Verhoeven EL, Prokop M, Moll FL, et al. Dynamic cine-computed tomography angiography imaging of standard and fenestrated endografts: differing effects on renal artery motion. Vasc Endovascular Surg 2008;42:25-31.

35) Muhs BE, Teutelink A, Prokop M, Vincken KL, Moll FL, Verhagen HJ. Endovascular aneurysm repair alters renal artery movement: a preliminary evaluation using dynamic CTA. J Endovasc Ther 2006;13:476-480.
36) Oshin OA, How TV, Brennan JA, Fisher RK, McWilliams RG, Vallabhaneni SR. Magnitude of the forces acting on target vessel stents as a result of a mismatch between native aortic anatomy and fenestrated stent-grafts. J Endovasc Ther 2011;18:569-575.

37) Post A, Diaz-Rodriguez P, Balouch B, Paulsen S, Wu S, Miller J, et al. Elucidating the role of graft compliance mismatch on intimal hyperplasia using an ex vivo organ culture model. Acta Biomater 2019;89:84-94. 